

\title{
A generalized entropy-based two-phase threshold algorithm for noisy medical image edge detection
}

\author{
A. Elaraby ${ }^{\mathrm{a}, \mathrm{b}}$ and D. Moratal ${ }^{\mathrm{b}, *}$ \\ a. Department of Mathematics, Faculty of Science, South Valley University, Qena, Egypt. \\ b. Center for Biomaterials and Tissue Engineering, Universitat Politècnica de València, Valencia, Spain. \\ Received 22 January 2016; received in revised form 5 July 2016; accepted 22 August 2016
}

\author{
KEYWORDS \\ Image edge detection; \\ Hill entropy; \\ Thresholding; \\ Canny edge detection; \\ Medical imaging; \\ Image analysis.
}

\begin{abstract}
Edge detection in medical imaging is a significant task for object recognition of human organs and is considered a pre-processing step in medical image segmentation and reconstruction. This article proposes an efficient approach based on generalized Hill entropy to find a good solution for detecting edges under noisy conditions in medical images. The proposed algorithm uses a two-phase thresholding: firstly, a global threshold calculated by means of generalized Hill entropy is used to separate the image into object and background. Afterwards, a local threshold value is determined for each part of the image. The final edge map image is a combination of these two separate images based on the three calculated thresholds. The performance of the proposed algorithm is compared to Canny and Tsallis entropy using sets of medical images corrupted by various types of noise. We used Pratt's Figure Of Merit (PFOM) as a quantitative measure for an objective comparison. Experimental results indicated that the proposed algorithm displayed superior noise resilience and better edge detection than Canny and Tsallis entropy methods for the four different types of noise analyzed, and thus it can be considered as a very interesting edge detection algorithm on noisy medical images.

(C) 2017 Sharif University of Technology. All rights reserved.
\end{abstract}

\section{Introduction}

In image analysis and computer vision systems, the edges of objects in an image contain important information that can be used as low-level features [1]. When noise exists in the image, an accurate detection of these contours becomes a very hard and timeconsuming task [2]. Producing the continuous contours of the object boundaries is a fundamental step in image processing and computer vision, especially in the field of feature detection and feature extraction [3], and it is the main goal of edge detection algorithms. The detection of these edges is a critical pre-processing step

*. Corresponding author. Tel.: +34 96387ro07, Ext. 88939 E-mail address: dmoratal@eln.upv.es (D. Moratal) for a variety of tasks, including object recognition $[4,5]$ and segmentation [6-9].

An edge can be defined as a boundary that divides an area of an image into two regions [1] or a single pixel with a local discontinuity in intensity [2]. So, different algorithms of edge detection can select the edges in various ways of representation, and the goodness or appropriateness of them will depend on the definition of those edges.

Edge detection is widely used in medical diagnosis [10] and all different medical imaging modalities (X-rays [11], ultrasonography [12], computed tomography [13], magnetic resonance imaging [14], nuclear medicine [15] or microscopy [16]).

The type of noise that can be encountered in all these different medical imaging modalities can be very different (salt \& pepper noise [17], additive white Gaussian noise [18], Poisson noise [19] or speckle 
noise [20]), and since none of the existing methods produces the optimum results for all images with different types of noise, finding an optimal method for edge detection is still an active field of research. Therefore, a robust algorithm is required to overcome the noise and consider the global structure of the edges to reduce broken ones.

Entropy is a concept in information theory which is used to measure the amount of information in a message [21-25]. Entropy is defined in terms of the probabilistic behavior of a source of information. Compared with other methods, the most important advantages of entropy-based approaches for edge detection are the ease of its implementation and the few number of operations [26]. Entropy-based approaches are very stable and efficient in noisy environments [26]. Their comparison with other edge-detection algorithms has shown that they have high capability and have been successfully applied to problems of edge detection in noisy image [26].

This article presents a new edge detection algorithm based on generalized Hill entropy for noisy images with the goal of extracting continuous edges and reducing the number of broken ones by means of a two-phase thresholding (see Figure 1 for an schematic overview of the presented algorithm).

The structure of this paper is as follows: Section 2 introduces the related work to edge detection algorithms; Section 3 presents the proposed edge detection approach based on generalized Hill entropy;
Section 4 contains experiments and Section 5 contains results and discussion.

\section{Related work}

Many edge detection algorithms have been proposed. The results are usually examined either by visual inspection as a qualitative measure $[1,26]$ or quantitatively by different indexes [27-35]. Some of these algorithms utilize a linking technique collecting pixels that belong to a set of edges [36-38]. In this context, the linking technique cannot be perfect except for simple shapes like lines or circles [36]; the use of image filters presents drawback which may affected image features, and thus the localization ability of an edge detector becomes poor [39].

Recently, many papers have been published in the area of image edge detection [27-35] that tests its importance as follows: Lopez-Molina et al. [27] presented a study that focuses on the improvements of edge detection by using Anisotropic Diffusion (AD) instead of Gaussian Linear Filtering (GLF); in [28], the modified scheme is presented to improve the performance of traditional Canny edge detection through an adaptive filter based on bi-dimensional general auto-regression model; in [29], Lopez-Molina et al. presented a novel edge detection framework based on the measurement of grey level changes using a new class of functions called relief functions; in [30], a new edge detection method that combines smoothing spline algorithm and gray-

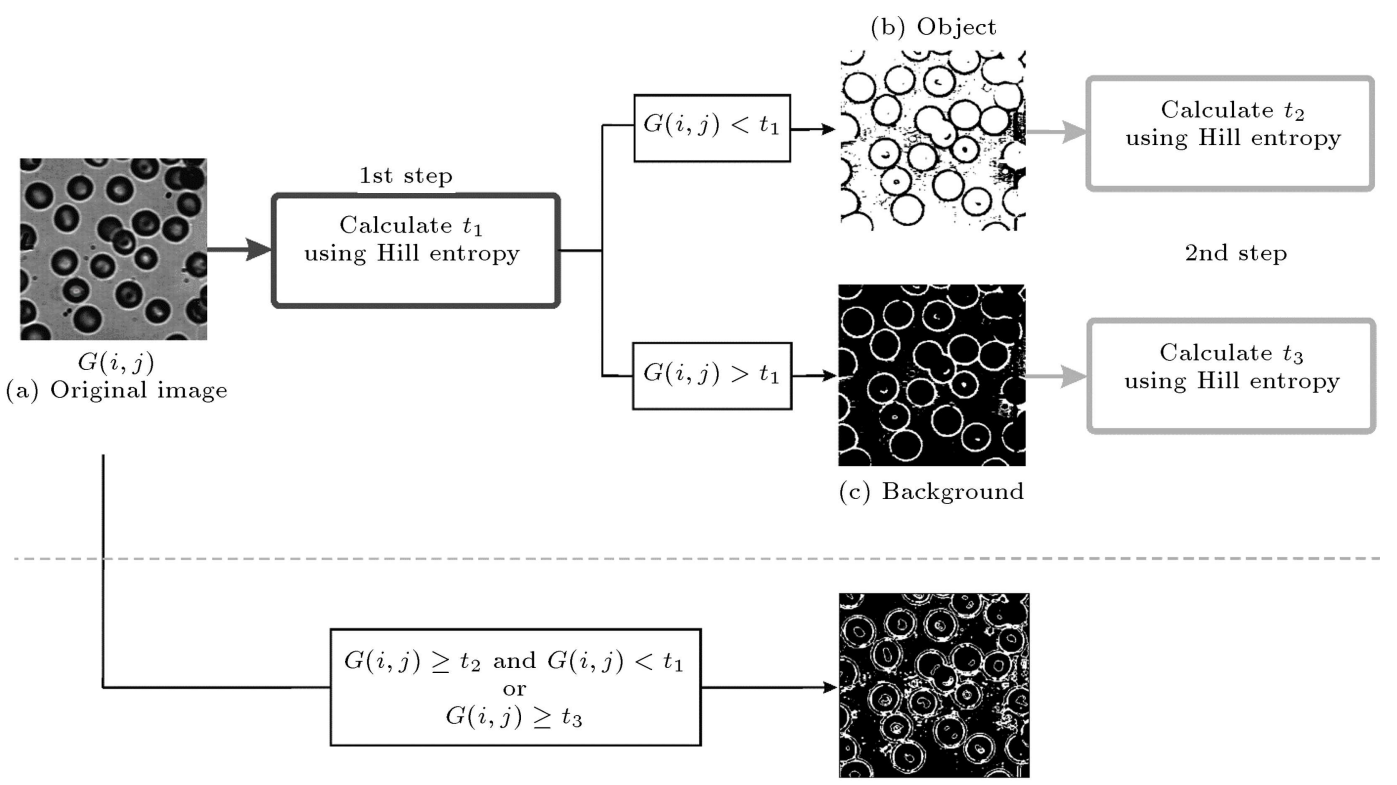

(d) Final edge map

Figure 1. Schematic overview of the proposed algorithm where different steps can be observed. Original image (a) is divided into two parts (object (b) and background (c)) following the threshold value, $t_{1}$, calculated by means of Hill entropy (1st step of the algorithm). Afterwards, the threshold values $t_{2}$ and $t_{3}$ are calculated from object (b) and background (c), respectively, by Hill entropy (the 2 nd step of the algorithm). The final edge map (d) is obtained by applying the threshold values $t_{1}, t_{2}$, and $t_{3}$ to the original image. 
moments operator is introduced; in [31], Ray presented a scheme for unsupervised edge detection that uses the highly efficient Absolute Difference Mask (ADM) algorithm to generate the initial edge image and uses a subsequent modified non-maximal suppression scheme to optimize the edge output resulting in the final edge map; in [32], Lopez-Molina et al. presented a multiscale method for edge detection based on increasing Gaussian smoothing, the Sobel operators, and coarseto-fine edge tracking; in [33], a study focusing on the edge detection process by the generation of a fuzzy representation of the edges is shown; in [34], a new approach is presented for edge detection using a combination of Bacterial Foraging Algorithm (BFA) and a probabilistic derivative technique derived from Ant Colony Systems; in [35], a new PSO-based approach is presented to detect edges in noisy images and reduce the broken and jagged ones by means of a developed penalized fitness function based on the possibility score of a curve fitted on an edge and its curvature cost.

Every edge detection algorithm has its advantages and disadvantages and does not appear to be an optimal edge detector that could be able to detect the edges of any image type and show high resistance to noise. Therefore, efficient algorithms are required to explore edge detection under challenging conditions with high resistance to noise, reducing the shortcomings of traditional edge detectors at the same time.

Some of the most popular edge detectors are Sobel [40] and Prewitt [41] based on the first-order derivative of the pixel intensities or the Laplacian-ofGaussian (LoG) $[42,43]$ edge detector that uses instead the second-order differential operators to detect the location of edges. However, these algorithms tend to be sensitive to noise, which is actually a high frequency phenomenon. In 1986, Canny [44] proposed an edge detector, which combines a smoothing function with zero-crossing-based edge detection, resulting into an algorithm more resilient to noise than the previously mentioned ones.

Entropy is an uncertainty measure introduced by Shannon into information theory to describe how much information is contained in a source governed by a probability law [23], and it has played an important role in recent work on edge detection algorithms: in [45], Xiao et al. proposed a Gray-Level \& Gradient Magnitude (GLGM) histogram for thresholding. GLGM histogram employs Fibonacci quantized gradient magnitude to effectively characterize spatial information by applying entropic image thresholding; Singh and Singh [26] proposed an algorithm based on Shannon entropy for edge detection in gray level images obtaining acceptable results; in [46], El-Khamy et al. used the relationship of the probability partition and the fuzzy 2-partition of the image gradient to select the optimal gradient-threshold, then it selects the algorithm that assures that the entropy reaches a minimum value; in [47], El-Sayed, presented a new algorithm for edge detection using both Shannon entropy and Tsallis entropy and in [48], Elaraby et al. proposed a new algorithm for edge detection of images based on hybrid types of entropy.

\section{The proposed approach based on generalized hill entropy}

In this section, we discuss the generalized Hill entropy by which our proposed edge-detection algorithm is based. Let $p_{1}, p_{2}, \ldots, p_{k}$ be the probability distribution of a discrete source. Therefore, $0 \leq p_{i} \leq 1, i=$ $1,2, \ldots, k$, and $\sum_{i=1}^{k} p_{i}=1$, where $k$ is the total number of states. The entropy of a discrete source is often obtained from the probability distribution.

The Shannon entropy [23] is defined as follows:

$$
H(p)=-\sum_{i=1}^{k} p_{i} \ln \left(p_{i}\right) .
$$

This formalism has been shown to be restricted to the domain of validity of the Boltzmann-Gibbs-Shannon (BGS) statistics. These statistics seem to describe nature when effective microscopic interactions and microscopic memory are short-ranged. Generally, systems that comply with BGS statistics are called extensive systems. If the physical system can be decomposed into two statistically independent subsystems $A$ and $B$, the probability of the joined system is $p^{A+B}=p^{A} \cdot p^{B}$. It has been verified that the Shannon entropy has the extensive property (additive) [23]:

$$
H(A+B)=H(A)+H(B) .
$$

The generalized entropy of Hill [49-51] is defined as:

$$
N_{\alpha}=\left(\sum_{i=1}^{W} p_{i}^{\alpha}\right)^{\frac{1}{1-\alpha}}, \text { for } \alpha \geq 0 \text { and } \alpha \neq 1
$$

Hill entropy is non-extensive in such a way that for a statistical independent system, the entropy of the system is defined by the following pseudo additive entropic rule:

$$
N_{\alpha}(A+B)=N_{\alpha}(A)+N_{\alpha}(B)+(1-\alpha) \cdot N_{\alpha}(A) \cdot B_{\alpha}(B) .
$$

The concept of entropy becomes increasingly important in image processing, when an image can be interpreted as an information source with the probability law given by its image histogram [52-56].

For an image with $k$ gray-levels, let $p_{1}, p_{2}, \ldots$, $p_{t}, p_{t+1}, \ldots, p_{k}$ be its probability distribution, where $p_{t}$ is the normalized histogram (i.e., $p_{t}=h_{t} /((M \times N))$ ) 
and $h_{t}$ is the gray level histogram. Using this distribution, we can derive two probability distributions, one for the object (class $A$ ) and the other for the background (class $B$ ) as follows:

$$
\begin{aligned}
& p_{A}: \frac{p_{1}}{P_{A}}, \frac{p_{2}}{P_{A}}, \ldots, \frac{p_{t}}{P_{A}}, \\
& p_{B}: \frac{p_{t+1}}{P_{B}}, \frac{p_{t+2}}{P_{B}}, \ldots, \frac{p_{k}}{P_{B}}, \\
& P_{A}=\sum_{i=1}^{t} p_{i}, \quad P_{B}=\sum_{i=t+1}^{k} p_{i},
\end{aligned}
$$

where $t$ is the threshold value.

In terms of the definition of Hill entropy, the entropy of object pixels $\left(N_{\alpha}^{A}\right)$ and background pixels $\left(N_{\alpha}^{B}\right)$ can be defined as follows:

$$
\begin{aligned}
& N_{\alpha}^{A}=\sum_{i=1}^{W}\left(\frac{p_{i}^{\alpha}}{P_{A}}\right)^{\frac{1}{1-\alpha}} \\
& N_{\alpha}^{B}=\sum_{i=1}^{W}\left(\frac{p_{i}^{\alpha}}{P_{B}}\right)^{\frac{1}{1-\alpha}} .
\end{aligned}
$$

Hill entropy, $N_{\alpha}(t)$, is parametrically dependent upon threshold value $t$ for the object and the background. It is formulated as the sum of each entropy, allowing the pseudo-additive property for statistically independent systems, as in Eq. (4). We try to maximize information measure between the two classes (object and background). When $N_{\alpha}(t)$ is maximized, luminance level $(t)$ that maximizes the function is considered to be the optimum threshold value. This can be done with low-computational effort:

$$
\begin{aligned}
t^{\mathrm{opt}}= & \operatorname{Arg} \max \left[N_{\alpha}^{A}(t)+N_{\alpha}^{B}(t)+(1-\alpha)\right. \\
& \left.. N_{\alpha}^{A}(t) \cdot N_{\alpha}^{B}(t)\right]
\end{aligned}
$$

when $\alpha \rightarrow 1$, the threshold value in Eq. (8) equals the same value found by Shannon entropy. Thus, this proposed method includes Shannon's method as a special case. The following expression can be used as a criterion function to obtain the optimal threshold when $\alpha \rightarrow 1$ :

$$
t_{S h}^{\mathrm{opt}}=\operatorname{Arg} \max \left[N_{\alpha}^{A}(t)+N_{\alpha}^{B}(t)\right]
$$

where $t_{S h}^{\mathrm{opt}}$ is the Shannon entropy.

By using a spatial filtering mask of size $3 \times 3$ (the smallest possible meaningful size), the probability of each central pixel of the window can be determined by entropy as $H(C p i x)=-p_{c} \ln \left(p_{c}\right)$, where $p_{c}$ is the probability of central pixel $C p i x$ of the binary image under the window.

When the probability of central pixel $p_{c}=1$, then the entropy of that pixel equals zero. Thus, if the gray level of all pixels under the window is homogeneous, $p_{c}=1$ and $H=0$. In this situation, the central pixel is not an edge pixel. At $p_{c}=7 / 9$ or $p_{c}=8 / 9$, the variety for gray level of pixels under the window is low. For these cases, the central pixel is not considered an edge pixel. In the remaining cases, where $p_{c} \leq 6 / 9$, the variety of gray level of pixels under the window is high, and thus we can assume that we are on an edge pixel.

The proposed algorithm based on generalized hill entropycan be summarized as follows:

1. Input: A digital gray-scale image $I$ of size $M \times N$.

2. Let $f(a, b)$ be the original gray value of the pixel at point $(a, b)$ :

$$
a=1,2, \ldots, M, b=1,2, \ldots, N .
$$

3. Calculate the probability distribution $p_{i}, 0 \leq i \leq$ 255 for every image pixel.

4. For all $t \in\{0,1, \ldots, 255\}$ :

4.1. Calculate $P_{A}, P_{B}, p_{A}$, and $p_{B}$ as:

$$
\begin{aligned}
& p_{A}: \frac{p_{1}}{P_{A}}, \frac{p_{2}}{P_{A}}, \ldots, \frac{p_{t}}{P_{A}}, \\
& p_{B}: \frac{p_{t+1}}{P_{B}}, \frac{p_{t+2}}{P_{B}}, \ldots, \frac{p_{z}}{P_{B}}, \\
& P_{A}=\sum_{i=1}^{t} p_{i}, \quad \text { and } \quad P_{B}=\sum_{i=t+1}^{z} p_{i} .
\end{aligned}
$$

4.2. Calculate the optimum threshold value $t^{*}$ as:

$$
t^{*}=\operatorname{Arg} \max \left[N_{\alpha}^{A}(t)+N_{\alpha}^{B}(t)+(1-\alpha) \cdot N_{\alpha}^{A}(t) \cdot N_{\alpha}^{B}(t)\right] .
$$

5. Create a binary image: For all $a, b$ :

$$
\text { If } I(a, b) \leq t^{*} \text { then } f(a, b)=0 \text { else } f(a, b)=1 \text {. }
$$

6. Create a mask, $S$, with $3 \times 3, \beta=(m-1) / 2$ and $\gamma=(n-1) / 2$.

7. Create an $M \times N$ output image, $g$ for all $a$ and $b$, set $g(a, b)=f(a, b)$.

8. Checking for edge pixels:

For all $b \in\{\gamma+1, \ldots, N-\gamma\}$, and

$a \in\{\beta+1, \ldots, M-\beta\}$, sum $=0$.

For all $k \in\{-\gamma, \ldots, \gamma\}$, and $j \in\{-\beta, \ldots, \beta\}$, If $f(a, b)=f(a+j, b+k)$, then sum $=$ sum +1 . If $($ sum $>6)$, then $g(a, b)=0$; else, $g(a, b)=1$.

9. Output: The edge detection image $g$ of $I$. 


\subsection{Time complexity}

Time complexity of the proposed approach is $O(M \times$ $N \times m \times n)$, where $M \times N$ is the number of pixels in $\mathbf{I}$, and $m \times n$ is the number of pixels in $w$ 's window. As can be observed, time complexity for computing each threshold values $t_{1}, t_{2}$, and $t_{3}$ is linear with the number of pixels. The reason is that computing the gray-level histogram of $I$ takes $O(M \times N)$, and finding $t^{\text {opt }}$ takes $O\left(k^{2}\right)$, where $k$ is the number of gray-level in $I$. Since $k^{2} \ll M \times N, O\left(M \times N+k^{2}\right)$ leads to $O(M \times N)$. The algorithm shows that building the binary image is also linear in the number of pixels, i.e. $O(M \times N)$. Finally, it is well-known that the time complexity of the application of a spatial discrete filter of order $m \times n$ in an $M \times N$ image can be done in $O(M \times N \times m \times n)$. As a result, this last step dominates the time complexity of the process, i.e. $O(4 \times M \times N+M \times N \times m \times n)$ leads to $O(M \times N \times m \times n)$.

The overall time complexity of Canny edge detector is $O(M \times N \log [M \times N])$. Thus, the computational complexity of the proposed solution is asymptotically equivalent to the simplest techniques.

\section{Experimental design}

All the analyses were performed using MATLAB R2012b (The Mathworks, Inc., Natick, MA, USA) running on an Intel Core ${ }^{\mathrm{TM}} 2$ Duo $2.20 \mathrm{GHz}$ personal computer with $3 \mathrm{~GB}$ of RAM.

To investigate the new algorithm effectiveness, we have compared it to Canny algorithm, as it is considered the optimal edge detector [57], and to Tsallis entropy, as it has, theoretically, similar properties. The three algorithms have been applied to different medical images with various types of noise. This section also describes image sets and performance measurements that have been used in the experiments.

\subsection{Image sets}

Image set includes one sagittal and one axial $\mathrm{T} 1$ weighted Magnetic Resonance (MR) images of a human brain, one axial $\mathrm{T} 1$ weighted MR image of an intervertebral disc, and one sagittal MR image of a whole human spine (see Figure 2). To explore the performance of the new algorithm in noisy environments, these images are corrupted by four types of noise: salt \& pepper (noise density $=0.05$ ), Gaussian (zero mean noise with variance $=0.01$ ), Poisson, and speckle (uniformly distributed random noise with mean $=0$ and variance $=0.04$ ) noise.

\subsection{Quantitative performance measurement}

The performance of the edge detection on noisy images is evaluated by comparing the result provided by



Original image
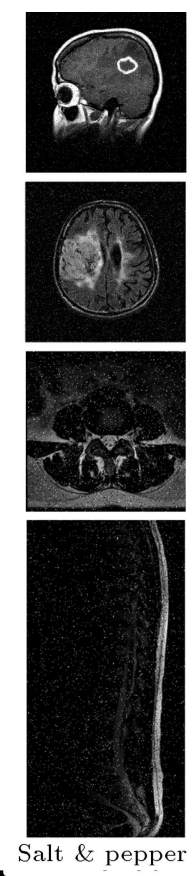
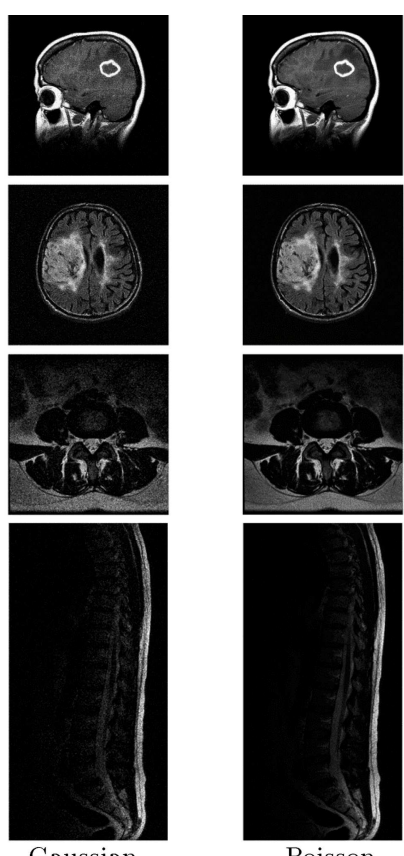

Gaussian

Noisy images



Poisson


Speckle

Figure 2. Medical images used in this work to test the performance of the proposed algorithm compared with that of Canny and Tsallis entropy edge detection algorithms. These original images (the first left column) were corrupted by various types of noise (salt \& pepper, Gaussian, Poisson and speckle): (a) Sagittal T1 weighted MR image of a human brain, (b) axial T1 weighted MR image of a human brain, (c) axial T1 weighted MR image of an intervertebral disc, and (d) sagittal MR image of a whole human spine. 
Canny and Tsallis entropy with that of our proposed method on the same images without noise, considered as reference for the optimal edge detection.

For an objective comparison of the localization accuracy of the edge detection algorithms, Pratt's Figure Of Merit (PFOM) is used as a quantitative measure [58]. PFOM is defined as:

$$
R=\frac{1}{\max \left(I_{I}, I_{A}\right)} \sum_{i=1}^{I_{A}} \frac{1}{1+\beta d(i)^{2}}
$$

where $I_{I}$ and $I_{A}$ indicate the number of ideal and actual edge points in ideal edge map and the generated edge map images, $d(i)$ is the distance between pixel $i$ in the generated edge map and the nearest ideal edge point in the ideal edge map, and $\beta$ is a constant scale factor which is typically set to $1 / 9$. This measure is an index to compute the localization accuracy of edge detection algorithms. $A$ larger value of $R$ indicates stronger performance.

\section{Results and discussion}

This section presents the results and discussion of the comparison (qualitatively and quantitatively) of the proposed algorithm with Canny and Tsallis entropy algorithms.
The results obtained from these three methods are included in Subsection 5.1 for a visual analysis and in Subsection 5.2 for a quantitative analysis. The results of each edge map depend on the thresholding values selected by means of the generalized Hill entropy for that image under different noise types. Table 1 displays the values of three thresholds for all four images (for the original image and for the four different added types of noise).

\subsection{Subjective comparison}

For a qualitatively comparison of our proposed algorithm and Canny and Tsallis entropy, we applied the three algorithms to the images previously described as corrupted by different types of noise. The resulting edge maps are shown in Figure 3.

The resulting images show that the proposed algorithm performed better than Canny and Tsallis entropy on the studied set of images. The boundary of the objects is more clearly defined after applying our algorithm than using Canny or Tsallis entropy algorithms for edge detection, and this shows that the proposed algorithm is resistant to noise. Canny algorithm, even with post-processing, did not work well for these noisy images and many noise spots in the resulting images were observed.

Table 1. Threshold values selected by generalized Hill entropy for the different analyzed images (for the original image and for the four different added types of noise). Image 1: sagittal T1 weighted MR image of a human brain; Image 2: axial T1 weighted MR image of a human brain; Image 3: axial T1 weighted MR image of an intervertebral disc; and Image 4: sagittal MR image of whole human spine.

\begin{tabular}{|c|c|c|c|c|c|}
\hline & Original & Salt \& pepper & Gaussian & Poisson & Speckle \\
\hline \multicolumn{6}{|c|}{ Image 1} \\
\hline$t_{1}$ & 138 & 131 & 137 & 141 & 142 \\
\hline$t_{2}$ & 83 & 78 & 68 & 87 & 88 \\
\hline$t_{3}$ & 191 & 185 & 189 & 95 & 188 \\
\hline \multicolumn{6}{|c|}{ Image 2} \\
\hline$t_{1}$ & 125 & 119 & 122 & 126 & 142 \\
\hline$t_{2}$ & 68 & 64 & 63 & 60 & 75 \\
\hline$t_{3}$ & 209 & 162 & 191 & 93 & 197 \\
\hline \multicolumn{6}{|c|}{ Image 3} \\
\hline$t_{1}$ & 89 & 88 & 116 & 95 & 117 \\
\hline$t_{2}$ & 46 & 46 & 61 & 50 & 60 \\
\hline$t_{3}$ & 160 & 151 & 189 & 175 & 197 \\
\hline \multicolumn{6}{|c|}{ Image 4} \\
\hline$t_{1}$ & 88 & 75 & 106 & 62 & 98 \\
\hline$t_{2}$ & 45 & 40 & 57 & 46 & 49 \\
\hline$t_{3}$ & 162 & 143 & 181 & 172 & 173 \\
\hline
\end{tabular}






Figure 3. Resulting edge map images obtained by applying the proposed algorithm and Canny and Tsallis entropy edge detection algorithms: $(a, b, c, d)$ Results of the edge detection algorithms applied to the four images studied in this work. For each image, the top row (.1) shows the results after applying the proposed algorithm for edge detection, the central row (.2) shows the results after applying Tsallis entropy algorithm, while the bottom row (.3) shows the results after applying Canny algorithm edge detection. Results are shown for the original images and for the same images after having been corrupted by salt \& pepper, Gaussian, Poisson, and speckle noise.

\subsection{Objective comparison}

To quantitatively compare the new algorithm with the Canny and Tsallis entropy algorithms, the localization accuracy was calculated using Pratt's Figure Of Merit
Table 2. Objective results (PFOM: Pratt's Figure Of Merit) for the proposed algorithm versus Tsallis entropy and Canny for all four images for the different added types of noise. Image 1: sagittal T1 weighted MR image of a human brain; Image 2: axial T1 weighted MR image of a human brain; Image 3: axial T1 weighted MR image of an intervertebral disc; Image 4: sagittal MR image of a whole human spine.

\begin{tabular}{cccc}
\hline & \multicolumn{3}{c}{ PFOM } \\
\cline { 2 - 4 } & $\begin{array}{c}\text { Proposed } \\
\text { algorithm }\end{array}$ & $\begin{array}{c}\text { Tsallis } \\
\text { entropy }\end{array}$ & Canny \\
\hline Image 1 & & & \\
Salt \& pepper & 68.3631 & 64.2356 & 37.0105 \\
Gaussian & 75.0607 & 70.5479 & 56.0638 \\
Poisson & 92.1007 & 90.1235 & 88.2085 \\
Speckle & 77.3533 & 71.5478 & 73.0216
\end{tabular}

$\begin{array}{cccc}\text { Image 2 } & & & \\ \text { Salt \& pepper } & 57.7091 & 52.8796 & 40.0925 \\ \text { Gaussian } & 72.7233 & 70.5487 & 34.6264 \\ \text { Poisson } & 96.6391 & 92.4593 & 89.0899 \\ \text { Speckle } & 84.4332 & 81.4568 & 79.6043\end{array}$

$\begin{array}{cccc}\text { Image 3 } & & & \\ \text { Salt \& pepper } & 63.7627 & 60.1236 & 51.5896 \\ \text { Gaussian } & 69.7806 & 63.8745 & 52.7855 \\ \text { Poisson } & 94.7062 & 92.9658 & 85.7877 \\ \text { Speckle } & 90.6569 & 86.7845 & 77.4794\end{array}$

\begin{tabular}{cccc} 
Image 4 & & & \\
Salt \& pepper & 51.1280 & 48.5892 & 40.2650 \\
Gaussian & 64.8006 & 60.8754 & 48.5146 \\
Poisson & 96.6102 & 91.4583 & 89.8145 \\
Speckle & 94.9092 & 92.4569 & 88.9649 \\
\hline
\end{tabular}

(PFOM) to the resulting images after applying different types of noise.

For the test images, we see the value of PFOM in Table 2. A high value of PFOM means that most of the edges are detected (better performance). The best performance is achieved when the images suffer from Poisson noise, followed by speckle noise and Gaussian noise. The worst performance of the proposed algorithm is achieved when the test images suffer from salt \& pepper noise. The same occurs for the Canny and Tsallis entropy algorithms, and these results repeat for all the images analyzed in this work.

From the visual and quantitative results, we can conclude that the proposed algorithm is competitive compared to the Canny and Tsallis entropy methods in terms of average performance. Furthermore, the 
proposed algorithm produces a better solution than Canny and Tsallis entropy for edge detection for every type of image under all types of noise, which indicates its potential and reliability. We infer that the proposed algorithm can be a very interesting option for problemspecific edge detection of medical images under noisy conditions.

\section{Conclusion}

In this article, a novel algorithm for detecting edges in medical images was presented. A 2-phase thresholding technique based on generalized Hill entropy was used to estimate threshold values required for the proposed algorithm. The first phase permitted us to determine a global threshold value that divides the image into two parts, called object and background. In the second step, a local threshold value was determined for each part of the image, merging the results in the final stage to get the edge map for the image. The proposed algorithm was examined and compared with Canny and Tsallis entropy algorithms on different medical images with four different types of noise. Subjective and objective measures to determine accuracy were used for a comparison of this algorithm. Experimental results showed that the proposed edge detector is more robust under noisy conditions than Canny and Tsallis entropy for all presented images under every type of noise.

\section{Acknowledgements}

The first author thanks Erasmus Mundus Action 2 WELCOME Program Third Call under Grant \#WELC1104034.

This work was supported in part by the Spanish Ministerio de Economía y Competitividad (MINECO) and by FEDER funds under Grant BFU2015-64380C2-2-R.

\section{References}

1. Umbaugh, S.E., Computer Imaging: Digital Image Analysis and Processing, CRC Press, Boca Raton (2005).

2. Gonzalez, R.C. and Woods, R.E., Digital Image Processing, Prentice Hall, New Jersey (2008).

3. Umbaugh, S.E., Digital Image Processing and Analysis: Human and Computer Vision Applications with CVIP Tools, 2nd Edn., CRC Press, Boca Raton (2011).

4. Ullman, S. and Basri, R. "Recognition by linear combinations of models", IEEE T Pattern Anal., 13, pp. 962-1006 (1991).

5. Ferrari, V., Fevrier, L., Jurie, F. and Schmid, C. "Groups of adjacent contour segments for object detection", IEEE T Pattern Anal., 30, pp. 36-51 (2008).
6. Malik, J., Belongie, S., Leung, T. and Shi, J. "Contour and texture analysis for image segmentation", Int. J. Comput. Vision., 43, pp. 7-27 (2001).

7. Arbelaez, P., Maire, M., Fowlkes, C. and Malik, J. "Contour detection and hierarchical image segmentation", IEEE T. Pattern Anal., 33(5), pp. 898-916 (2011).

8. Kass, M., Witkin, A. and Terzopoulos, D. "Snakes: Active contour models", Int. J. Comput. Vision., 1, pp. 321-331 (1988).

9. Azad, P., Gockel, T. and Dillmann, R., Computer Vision: Principles and Practice, Elektor International Media BV, Netherlands (2008).

10. McAuliffe, M.J., Lalonde, F.M., McGarry, D., Gandler, W., Csaky, K. and Trus. B.L. "Medical image processing, analysis \& visualization in clinical research, computer-based medical systems", Proceedings 14 th IEEE Symposium on Bethesda, MD, pp. 381-386 (2001).

11. Peng, T.T. "Detection of femur fractures in X-ray images", MSc Thesis, National University of Singapore (2002).

12. Nes, P.G. "Fast multi-scale edge-detection in medical ultrasound signals", Signal Process, 92, pp. 2394-2408 (2012).

13. Ontiverosa, S., Yagüea, J.A., Jiménez, R. and Broseda, F. "Computer tomography 3D edge detection comparative for metrology applications", Procedia Engineering, 63, pp. 710-719 (2013).

14. Tang, H., Wua, E.X., Ma, Q.Y., Gallagher, D., Perera, G. and Zhuang, T. "MRI brain image segmentation by multi-resolution edge detection and region selection", Comput. Med. Imag. Grap., 24, pp. 349-357 (2000).

15. Wang, C.X., Small, L., Snyde, W.E. and William, R. "Edge detection in gated cardiac nuclear medicine images, computer-based medical systems", ComputerBased Medical Systems, Proceedings 1994 IEEE Seventh Symposium on, Winston-Salem, NC, 10-12 June (1994).

16. Gebäck, T. and Koumoutsakos, P. "Edge detection in microscopy images using curvelets", BMC Bioinformatics, 10, p. 75 (2009).

17. Toprak, A. and Güler, I. "Impulse noise reduction in medical images with the use of switch mode fuzzy adaptive median filter", Digit Signal Process, 17, pp. 11-23 (2007).

18. Gravel, P., Beaudoin, G. and De Guise, J.A. "A method for modeling noise in medical images", IEEE T. Med. Imaging, 23, pp. 1221-1232 (2004).

19. Landi, G. and Piccolomini, E.L. "An efficient method for nonnegatively constrained Total Variation-based denoising of medical images corrupted by Poisson noise", Comput. Med. Imag. Grap., 36, pp. 38-46 (2012). 
20. Sánchez, M.G., Vidal, V., Verdú, G., Mayo, P. and Rodenas, F. "Medical image restoration with different types of noise", Proceedings of the 34th IEEE Annual International Conference EMBS, San Diego, California USA, 28 Aug. - 1 Sep. (2012).

21. Gray, R.M., Entropy and Information Theory, 1st Edn. Corrected, Springer (2013).

22. Shunsuke, I. "Information theory for continuous system", World Scientific, Singapore (1993).

23. Shannon, C.E. "A mathematical theory of communication", The Bell System Technical Journal, 27, pp. 379-423 (1948).

24. Léon, B., Science \& Information Theory, Dover Publications, 2nd Edn. (2004).

25. Pratt, W., Digital Image Processing, PIKS Scientific Inside, Wiley Interscience (2007).

26. Singh, B. and Singh, A.P. "Edge detection in gray level images based on the Shannon entropy", Journal of Computer Science, 4, pp. 186-191 (2008).

27. Lopez-Molina, C., Galara, M., Bustincea, H. and De Baets, B. "On the impact of anisotropic diffusion on edge detection", Pattern Recogn., 47, pp. 270-281 (2014).

28. Haoa, F., Shia, J., Zhanga, Z., Chenc, R. and Zhud, S. "Canny edge detection enhancement by general autoregression model and bi-dimensional maximum conditional entropy", Optik, 125, pp. 3946-3953 (2014).

29. Lopez-Molina, C., De Baets, B. and Bustince, H. "A framework for edge detection based on relief functions", Inform Sciences, 278, pp. 127-140 (2014).

30. Sun, Q., Hou, Y., Tan, Q., Li, C. and Liu, M. "A robust edge detection method with sub-pixel accuracy", Optik., 125, pp. 3449-3453 (2014).

31. Ray, K. "Unsupervised edge detection and noise detection from a single image", Pattern Recogn., 46, pp. 2067-2077 (2013).

32. Lopez-Molina, C., De Baets, B., Bustince, H., Sanz, J. and Barrenechea, E. "Multiscale edge detection based on Gaussian smoothing and edge tracking", KnowlBased Syst., 44, pp. 101-111 (2013).

33. Lopez-Molina, C., De Baets, B. and Bustince, H. "Generating fuzzy edge images from gradient magnitudes", Comput. Vis. Image. Und., 115, pp. 1571-1580 (2011).

34. Verma, O., Hanmandlu, M., Kumar, P., Chhabra, S. and Jindal, A. "A novel bacterial foraging technique for edge detection", Pattern Recogn Lett., 32, pp. 11871196 (2011).

35. Setayesh, M., Zhang, M. and Johnston, M. "A novel particle swarm optimisation approach to detecting continuous, thin and smooth edges in noisy images", Inform Sciences, 246, pp. 28-51 (2013).

36. Jevtic, A., Melgar, I. and Andina, D. "Ant based edge linking algorithm", Proceedings of 35th Annual Conference of the IEEE Industrial Electronics Society, Porto, Portugal, pp. 3353-3358 (2009).
37. Shih, F.Y. and Cheng, S. "Adaptive mathematical morphology for edge linking", Inform Sciences., 167, pp. 9-21 (2004).

38. Wei, L., Sheng, L., Yi, R.X. and Peng, D. "A new contour detection in mammogram using sequential edge linking", Second International Symposium on Intelligent Information Technology Application, Hong Kong, China, pp. 197-200 (2008).

39. Zhang, Y.D. and Wu, L.N. "Improved image filter based on SPCNN", Science in China Series F Information Sciences, 51(12), pp. 2115-2125 (2008).

40. Sobel, I. "Camera models and perception", Ph.D. Thesis, Stanford University, Stanford, CA (1970).

41. Prewitt, J. "Object enhancement and extraction", Picture Processing and Psychopictorics, B. Lipkin and A. Rosenfeld, Eds., New York: Academic, pp. 75-149 (1970).

42. Haralick, R.M. "Digital step edges from zero crossing of second directional derivatives", IEEE T Pattern Anal., 6, pp. 58-68 (1984).

43. Huertas, A. and Medioni, G. "Detection of intensity changes with subpixel accuracy using LaplacianGaussian masks", IEEE T Pattern Anal., 8, pp. 651664 (1986).

44. Canny, J. "A computational approach to edge detect", IEEE T Pattern Anal., 8, pp. 679-698 (1986).

45. Xiao, Y., Zhiguo, C. and Jansong, Y. "Entropic image thresholding based on GLGM histogram", Pattern Recogn Lett., 40, pp. 47-55 (2014).

46. El-Khamy, S.E., Galeb, I. and El-Yamany, N.A. "Fuzzy edge detection with minimum fuzzy entropy criterion", Proceedings 11th Mediterranean Electrotechnical Conference (IEEE), MELECON, pp. 498-503 (2002).

47. El-Sayed, A. "A new algorithm based entropic threshold for edge detection in images", International Journal of Computer Science Issues, 8, pp. 71-78 (2011).

48. Elaraby, A., El-Owny, H., Heshmat, M. and Abdel Rady, A.S. "Edge detection of noisy medical images based mixed entropy", Computer Engineering and Intelligent Systems, 4, pp. 97-106 (2013).

49. Hill, M.O. "Diversity and Evenness: A unifying notation and its consequences", Ecology, 54, pp. 427-432 (1973).

50. Jost, L. "Entropy and diversity", Oikos, 113, pp. 363375 (2006).

51. Evangelista, H.B.A., Thomaz, S.M., Mendes, R.S. and Evangelista, L.R. "Generalized entropy indices to measure $\alpha$ - and $\beta$ diversities of macrophytes", Braz. J. Phys., 39, pp. 396-401 (2009). 
52. Pun, T. "A new method for gray-level picture thresholding using the entropy of histogram", Signal Process, 2, pp. 223-237 (1980).

53. Kapur, J.N., Sahoo, P.K. and Wong, A.K.C. "A new method for gray-level picture thresholding using the entropy of histogram", Comput Vis Graph Image Process, 29, pp. 273-285 (1985).

54. Pal, N. and Pal, S. "Entropic thresholding", Signal Process., 16, pp. 97-108 (1989).

55. Chang, C.I., Chen, K., Wang, J. and Althouse, M. "A relative entropy-based approach to image thresholding", Pattern Recogn., 29, pp. 1275-1289 (1994).

56. Abutaleb, A.S. "Automatic thresholding of gray-level pictures using two-dimensional entropy", Comput Vis Graph Image Process, 47, pp. 22-32 (1989).

57. Shrivakshan, G.T. and Chandrasekar, C. "A comparison of various edge detection techniques used in image processing", International Journal of Computer Science Issues, 9, pp. 269-276 (2012).

58. Fu, W.O., Johnston, M. and Zhang, M. "Figure of merit based fitness functions in genetic programming for edge detection", Lecture Notes in Comput. Sci., 7673, pp. 22-31 (2012).

\section{Biographies}

Ahmed Elaraby works as Lecturer of Computer Science at the Faculty of Sciences, South Valley University in Egypt. He graduated in 2008 and worked as a Teaching Assistant at the same Faculty. Meanwhile, he received his $\mathrm{MSc}$ degree in the field of computer science in 2012 and his $\mathrm{PhD}$ in 2016. In 2012, he was granted for a 6-months a DAAD research scholarship at HTWG Konstanz University, Germany. In 2013, he was granted for a 6-month-research scholarship at
Caen University in France. In 2014, he was granted for a 6-month Erasmus Mundus research scholarship at Universitat Politècnica de València in Spain. In 2016, he was granted a one-month research visit at JINR in Russia. His main research interests are Image Computing, Interval Arithmetic, and Scientific Computing.

David Moratal is an Associate Professor of Electronics and Medical Imaging at the Universitat Politécnica de Valéncia (Spain) and Researcher at the Center for Biomaterials and Tissue Engineering of the same University, where he is the Director of the Biomedical Image Analysis Group. Dr. Moratal received his MSc (2001) and PhD (2006) degrees in Electrical and Electronics Engineering from the Universitat Politécnica de Valéncia, Spain. He is also Ingénieur Supélec (2001), from the École Supérieure d'Électricité, France. He has worked as a researcher in Magnetic Resonance Imaging (MRI) at Emory University School of Medicine, Atlanta, GA, USA, and has completed a Research Fellowship in MRI clinical applications at Hôpital Erasme, Brussels, Belgium. He has been Visiting Researcher working on microscopy image analysis of cerebral cortex at King's College London. His primary research interests include biomedical image analysis, medical image physics and acquisition, new technologies for data acquisition and reconstruction in magnetic resonance imaging, and the study of new methods and applications of medical imaging, with a special focus on the field of MRI. Dr. Moratal is coauthor of more than 80 scientific papers in the field of medical imaging and has published more than 300 contributions in international scientific meetings concerning different aspects of biomedical image analysis. He is coauthor of 10 chapter books and editor of 2 books. 